\title{
DEFORMATION THEORY OF FUCHSIAN EQUATIONS AND LOGARITHMIC CONNECTIONS
}

\author{
SZILÁRD SZABÓ
}

\begin{abstract}
Motivated by a remark and a question of Nicholas Katz, we characterize the tangent space of the space of Fuchsian equations with given generic exponents inside the corresponding moduli space of logarithmic connections: we construct a weight 1 Hodge structure on the tangent space of the moduli of logarithmic connections such that deformations of Fuchsian equations correspond to the $(1,0)$-part.
\end{abstract}

\section{Statement of Results}

Let $p_{1}, \ldots, p_{n} \in \mathbf{P}^{1}$ be $n \geq 2$ fixed points in the affine part of the complex projective line, and let $p_{0}$ be the point at infinity. Define $P$ to be the simple effective divisor $p_{0}+\cdots+p_{n}$ in $\mathbf{P}^{1}$, and let $P^{o}=$ $p_{1}+\cdots+p_{n}$. Consider the function

$$
\psi(z)=\left(z-p_{1}\right) \cdots\left(z-p_{n}\right)
$$

as an identification between $\mathcal{O}_{\mathbf{P}^{1}}$ and $\mathcal{O}_{\mathbf{P}^{1}}\left(-P^{o}\right)$ on the affine $\mathbf{A}^{1}=$ $\mathbf{P}^{1} \backslash\left\{p_{0}\right\}$.

For $w=w(z)$ a holomorphic function of the complex variable $z$, let $w^{(k)}$ stand for its $k$-th order (anti-holomorphic) differential with respect to $z$ and let

$$
w^{(m)}-\frac{G_{1}(z)}{\psi} w^{(m-1)}-\cdots-\frac{G_{m}(z)}{\psi^{m}} w=0,
$$

where the $G_{k}$ are polynomials in $z$, be a Fuchsian differential equation. Recall that this means that all the solutions $w$ grow at most polynomially with $\left(z-p_{j}\right)^{-1}$ near $p_{j}$ for any $1 \leq j \leq n$ (respectively, solutions grow at most polynomially with $z$ near infinity), in any given finite sector centered at the punctures. The left-hand side of (2) is a linear differential operator of the order $m$ of $w$, that we shall denote by $\mathcal{L}$. We shall identify the equation (2) with the operator $\mathcal{L}$. A classical result of I.L.Fuchs states that a necessary and sufficient condition on

Date: September 25, 2018.

Department of Geometry, Technical University, Egry J. u. 1/H, Budapest 1111, Hungary, szabosz@math.bme.hu. 
the degrees of the $G_{k}$ 's for $\mathcal{L}$ to be of Fuchsian type is that the degree of $G_{k}$ has to be at most $k(n-1)$.

Let us introduce the expressions

$$
\begin{aligned}
w_{1} & =w \\
w_{2} & =\psi \frac{\mathrm{d} w}{\mathrm{~d} z} \\
\vdots & \\
w_{m} & =\psi^{m-1} \frac{\mathrm{d}^{m-1} w}{\mathrm{~d} z^{m-1}} .
\end{aligned}
$$

We may think of $w$ as a local section of any line bundle $L$ over $\mathbf{P}^{1}$ fixed in advance, for instance the structure sheaf $\mathcal{O}$. Then, on the affine open $\mathbf{A}^{1}$ the function $w_{k}$ is meromorphic with zeroes of order at least $(k-1)$ in $P$. In other words, on the affine part $\mathbf{A}^{1}$ a vector $\left(w_{1}, \ldots, w_{m}\right)$ is a section of the holomorphic vector bundle

$$
\tilde{E}=\mathcal{O}_{\mathbf{P}^{1}} \oplus \mathcal{O}_{\mathbf{P}^{1}}\left(-P^{o}\right) \oplus \ldots \oplus \mathcal{O}_{\mathbf{P}^{1}}\left((1-m) P^{o}\right) .
$$

We equip $\tilde{E}$ with the algebraic integrable connection with logarithmic poles at $P$

$$
D_{\mathcal{L}}=\mathrm{d}^{1,0}-\frac{A(z)}{\psi(z)} \mathrm{d} z
$$

where $A(z)$ is the modified companion matrix

$$
A=\left(\begin{array}{cccccc}
0 & 0 & 0 & 0 & \ldots \ldots & G_{m} \\
1 & \psi^{\prime} & 0 & 0 & \ldots \ldots & G_{m-1} \\
0 & 1 & 2 \psi^{\prime} & 0 & \ldots \ldots & G_{m-2} \\
\vdots & \vdots & \vdots & & & \vdots \\
0 & 0 & 0 & \ldots & (m-2) \psi^{\prime} & G_{2} \\
0 & 0 & 0 & \ldots & 1 & G_{1}+(m-1) \psi^{\prime}
\end{array}\right)
$$

of equation (2). Here we have denoted $\psi^{\prime}=\mathrm{d} \psi / \mathrm{d} z$. One readily checks that a meromorphic function $w$ on some open set $U \subset \mathbf{A}^{1}$ with poles at most in $P$ locally solves (2) if and only if the vector $\left(w_{1}=w, w_{2}, \ldots, w_{m}\right)$ is a parallel section of $D_{\mathcal{L}}$ on $\tilde{E}$ over $U$ for some (hence, only one) vector $\left(w_{2}, \ldots, w_{m}\right)$.

Remark 1.1. Instead of the formulae (3), we could have simply chosen $w_{k}=w^{(k)}$, and the form of the same connection $D_{\mathcal{L}}$ in this trivialisation would then be a usual companion matrix. The reason for our choice for the extension (3) is that it gives rise to a logarithmic lattice. In fact, the two points of view are equivalent, so that a connection in modified companion form is also induced by an equation. 
By assumption, the integrable connection $D_{\mathcal{L}}$ is regular at infinity as well. Therefore, according to a theorem of N. Katz (Thm. II.1.12 [Del70]), there exists a lattice for the meromorphic bundle

$$
\mathcal{N}=\mathcal{O}_{\mathbf{P}^{1}}(* P) \oplus \ldots \oplus \mathcal{O}_{\mathbf{P}^{1}}(* P)
$$

at infinity with respect to which $D_{\mathcal{L}}$ is a logarithmic connection, i.e. its local form in any holomorphic trivialisation contains 1-forms with at most first-order poles. Such a lattice at infinity can be obtained similarly to the case of the other singularities. For this purpose, let $\zeta=$ $z^{-1}$ be a local coordinate at infinity. Recall that the first component $w=w_{1}$ of (3) is supposed to be a section of $\mathcal{O}_{\mathbf{P}^{1}}$. Then, a logarithmic lattice $\left(\tilde{w}_{1}, \ldots, \tilde{w}_{m}\right)$ at infinity can be obtained by the formulae

$$
\begin{aligned}
\tilde{w}_{1} & =w \\
\tilde{w}_{2} & =\zeta \frac{\mathrm{d} w}{\mathrm{~d} \zeta} \\
\vdots & \\
\tilde{w}_{m} & =\zeta^{m-1} \frac{\mathrm{d}^{m-1} w}{\mathrm{~d} \zeta^{m-1}},
\end{aligned}
$$

and the form of the connection is then again a modified companion matrix with entries in the last column equal to the coefficients of the equation multiplied by appropriate powers of $\zeta$ which make the connection logarithmic near infinity. Now one can check by induction that as a differential operator

$$
\zeta^{j} \frac{\mathrm{d}^{j}}{\mathrm{~d} \zeta^{j}}=(-z)^{j} \frac{\mathrm{d}^{j}}{\mathrm{~d} z^{j}}+\sum_{\kappa=0}^{j-1} a_{\kappa j}(-z)^{\kappa} \frac{\mathrm{d}^{\kappa}}{\mathrm{d} z^{\kappa}}
$$

for some integers $a_{\kappa j}, 0<\kappa<j<m-1$. Set then $a_{j j}=1$ and $a_{\kappa j}=0$ for $\kappa>j$. We see moreover that for large $z$ one has

$$
\psi(z) \approx z^{n}
$$

hence the trivialisations (3) and (6) are linked on $\mathbf{C}^{*}$ by the matrix

$$
B \operatorname{diag}\left(1,-z^{n-1}, \ldots,(-1)^{m-1} z^{(m-1)(n-1)}\right) A,
$$

with $A=\left(a_{\kappa j}\right)_{\kappa, j=1}^{m}$ and $B$ a holomorphic matrix on $\mathbf{P}^{1} \backslash\{0\}$, invertible at $\infty$. It follows that we defined a logarithmic extension of the connection $D_{\mathcal{L}}$ on the holomorphic bundle

$$
E_{\mathcal{L}}=\mathcal{O}_{\mathbf{P}^{1}} \oplus \mathcal{O}_{\mathbf{P}^{1}}\left(\infty-P^{o}\right) \oplus \ldots \oplus \mathcal{O}_{\mathbf{P}^{1}}\left((m-1)\left(\infty-P^{o}\right)\right)
$$

over $\mathbf{P}^{1}$. Hence, (3) induces a bijective correspondence between local solutions of (2) and local parallel sections of the logarithmic connection 
$D_{\mathcal{L}}$ on the holomorphic bundle $E_{\mathcal{L}}$. It is not difficult to see that had we started out with taking $w$ to be a section of a non-trivial line bundle $L$, we would have obtained a logarithmic connection on $E_{\mathcal{L}} \otimes L$. The following fundamental result is part of folklore; however, we give a proof in the Appendix for lack of appropriate reference.

Proposition 1.2. If the logarithmic connections induced by the Fuchsian equations $\mathcal{L}_{1}, \mathcal{L}_{2}$ are gauge-equivalent, then $\mathcal{L}_{1}=\mathcal{L}_{2}$.

Consider now the residue $\operatorname{res}\left(p, D_{\mathcal{L}}\right)$ of $D_{\mathcal{L}}$ on $E_{\mathcal{L}}$ at each of the singular points $p \in P$; it is a well-defined endomorphism of the fiber $\left(E_{\mathcal{L}}\right)_{p_{j}}$ of $E_{\mathcal{L}}$ at $p$, defined by applying $D_{\mathcal{L}}$ to the vector field $\left(z-p_{j}\right) \frac{\partial}{\partial z}$ and restricting to $\left(E_{\mathcal{L}}\right)_{p_{j}}$ for $0<j \leq n$ (respectively, $-z^{-1} \frac{\partial}{\partial z}$ and restricting to $\left.\left(E_{\mathcal{L}}\right)_{p_{0}}\right)$. Denote by $\left\{\mu_{1}^{j}, \ldots, \mu_{m}^{j}\right\}$ the set of generalized eigenvalues of the residue at $p_{j}$; this is also the set of exponents of the differential equation $\mathcal{L}$ at $p_{j}$ [Sza08].

Remark 1.3. Notice in particular that $\operatorname{deg}\left(E_{\mathcal{L}}\right)=(1-n) m(m-1) / 2$, in accordance with the classical Fuchs' relation, which states that the sum $\sum_{j, k} \mu_{k}^{j}$ of the eigenvalues of the residues of $D_{\mathcal{L}}$ in all singularities (including infinity) is equal to $(n-1) m(m-1) / 2$, and with the residue theorem which states that the sum of the eigenvalues of the residues must be minus the degree of $E_{\mathcal{L}}$.

Throughout the paper, we will assume the genericity conditions :

Condition 1.4. ((i)) The eigenvalues $\mu_{1}^{j}, \ldots, \mu_{m}^{j}$ of the residue of the integrable connection $D_{\mathcal{L}}$ at each singularity $p_{j}$ do not differ by integers. (In particular, they are distinct.) We call this the non-resonance condition.

((ii)) For any $k \in\{1, \ldots, m\}$ and any indices $\alpha_{l}^{j}$ for $j \in\{0, \ldots, n\}$ and $l \in\{1, \ldots, k\}$ the sum $\sum_{j=0}^{n} \sum_{l=1}^{k} \mu_{\alpha_{l}^{j}}^{j}$ is not an integer.

Condition (i) implies that all the residues $\operatorname{res}\left(p_{j}, D_{\mathcal{L}}\right)$ are regular semi-simple endomorphisms of the corresponding fibre $\left(E_{\mathcal{L}}\right)_{p_{j}}$. Condition (ii) says that there can be no proper $D$-invariant subbundles of $E$, because by the residue theorem the negative of the degree of such a subbundle would be a sum as in the condition. In particular, it follows that any logarithmic connection with these eigenvalues of its residue is stable.

Let us choose an arbitrary ordering of the eigenvalues $\mu_{1}^{j}, \ldots, \mu_{m}^{j}$, and denote the corresponding eigenvectors of $\operatorname{res}\left(p_{j}, D_{\mathcal{L}}\right)$ by $v_{1}^{j}, \ldots, v_{m}^{j} \in$ $\left(E_{\mathcal{L}}\right)_{p_{j}}$. For $0 \leq k \leq m$ let $E_{k}^{j}$ be the subspace of $\left(E_{\mathcal{L}}\right)_{p_{j}}$ spanned by 
the vectors $\left\{v_{k+1}^{j}, \ldots, v_{m}^{j}\right\}$. These subspaces define a full flag in $\left(E_{\mathcal{L}}\right)_{p_{j}}$ :

$$
\left(E_{\mathcal{L}}\right)_{p_{j}}=E_{0}^{j} \supset E_{1}^{j} \supset \cdots \supset E_{m-1}^{j} \supset E_{m}^{j}=\{0\} .
$$

In different terms, we get a quasi-parabolic strucutre, which is clearly compatible with $\operatorname{res}\left(p_{j}, D_{\mathcal{L}}\right)$ in the sense that the residue maps each term of the filtration into itself.

Furthermore, for any $j \in\{0, \ldots, n\}$ let us choose real numbers $\beta_{1}^{j}, \ldots, \beta_{m}^{j}$ such that $\beta_{k}^{j}$ and $\beta_{l}^{j}$ do not differ by an integer for any $j, k, l$ (in particular, they are distinct). We will call such a choice $\beta_{1}^{j}, \ldots, \beta_{m}^{j}$ generic parabolic weights.

We are interested in the following two numbers:

((i)) the dimension $e$ of the space $\mathcal{E}$ of deformations of the polynomials in (2) such that all residues of the associated integrable connection $D_{\mathcal{L}}$ remain in the same conjugacy class

((ii)) the dimension $c$ of the moduli space $\mathcal{M}$ of S-equivalence classes of $\beta$-stable integrable connections $(E, D)$ logarithmic in $P$ over a vector bundle $E$ of degree $d=(1-n) m(m-1) / 2$, with fixed conjugacy classes of all its residues.

Remark 1.5. There are various sources for the definition of the moduli space $\mathcal{M}$. In any case, it is a quasi-projective scheme which is smooth for generic choice of eigenvalues $\mu$. An analytic construction is carried out (in greater generality than what we need here, namely in the case with irregular singularities) in Sections 6-8 of [BB04]. In the $\mathrm{Sl}_{2}$-case an algebraic construction of $\mathcal{M}$ in the relative setting is given in [IS06], which is generalized in Ina06] to the higher-rank case. On the other hand, the space $\mathcal{E}$ is well-known to be an affine space, see e.g. [Inc26].

It is immediate that $e \leq c$, for the space of Fuchsian deformations (i) is contained in the space of integrable connections (ii) having the right monodromy, and by Proposition 1.2 this inclusion map is injective. In short, we will call deformations leaving invariant the conjugacy classes of all the residues locally isomonodromic. In the introduction of his book [Kat96], N. Katz computed $c$, and noticed the equality

$$
c=2 e \text {. }
$$

In fact, both sides of this equation turn out to be

$$
2-2 m^{2}+m(m-1)(n+1) .
$$

He also asked whether a geometric reason underlies this equality, more precisely, whether a weight 1 Hodge structure can be found on the 
tangent to the moduli space of integrable connections, whose $(1,0)$ part would give precisely the locally isomonodromic deformations of Fuchsian equations.

We will define such a Hodge structure in Section 2,

Theorem 1.6. Let $D_{\mathcal{L}}$ be an integrable connection (4)-(5) induced by a Fuchsian equation (2) satisfying Condition 1.4. Then there exists a natural weight 1 Hodge structure on the tangent at $D_{\mathcal{L}}$ to the moduli space $\mathcal{M}$ of integrable connections

$$
T_{D_{\mathcal{L}}} \mathcal{M}=H^{1,0} \oplus H^{0,1}
$$

with the property that its part of type $(1,0)$ is the tangent of the space $\mathcal{E}$ of locally isomonodromic deformations of the Fuchsian equation:

$$
T_{D_{\mathcal{L}}} \mathcal{E}=H^{1,0}
$$

The Hodge structure comes from a hypercohomology long exact sequence, and is well-defined on the tangent space at all elements of the moduli space. Notice that a similar exact sequence already appeared several times in the literature: for example, in Proposition 4.1 of [Nit93] as well as in Proposition 6.1 of [IIS06]. In addition, in the case of rank 2 Higgs bundles over a projective curve a similar splitting is used in Section 7 of Hit87 to obtain topological information on the moduli space.

Notice also that our result is completely algebraic and does not involve the choice of parabolic weights; it would be interesting to study the dependence of these results on the weight chambers. In the particular case of rank 2 bundles with 4 parabolic points this is carried out in [LSS10].

In Subsection 2.3, we draw the following consequence of our Theorem:

Corollary 1.7. $\mathcal{E}$ is an algebraic subvariety which is Lagrangian with respect to the natural holomorphic symplectic structure of the de Rham moduli space.

We are aware that J. Aidan [Aid has results in the same direction. There are quite a few possible directions to generalise our results. First, one could study the same questions on moduli spaces of integrable connections on higher-genus curves with marked points - we plan to treat this question in a forthcoming paper [SS]. Similar structures for an arbitrary reductive structure group on higher-genus curves (but without logarithmic points) called opers were defined by A. Beilinson and V. Drinfeld in BD05]. This could possibly lead to a generalisation 
for other structure groups too. Another possible generalisation would be to allow for arbitrary residues.

Notice finally that a Dolbeault analog of our embedding of the space of Fuchsian equations to the moduli space appeared in the article of $\mathrm{N}$. Hitchin [Hit92] under the name Teichmüller component.

Acknowledgments. During the preparation of this text the author benefited of discussions with D. Bertrand, O. Biquard, P. Boalch, T. Hausel, N. Hitchin, M-H. Saito, C. Simpson, A. Stipsicz and A. Szenes; we would like to thank for the insightful comments and motivating discussions. The paper was written with the support of Hungarian Science Foundation OTKA grant NK 81203 and Lendület project.

\section{The Hodge structure}

2.1. Construction. First, let us recall some basic facts about deformations of logarithmic connections on curves. Most of this material is proved in Section 12 of Biq97 for projective varieties with a smooth polar divisor.

Let $(E, D)$ be an arbitrary element of $\mathcal{M}$. Let us denote by $\mathcal{E} n d_{\text {par }}(E)$ the sheaf of parabolic endomorphisms: by definition, a section $\varphi$ near

$p_{j} \in P$ of the endomorphism-bundle of $E$ is parabolic if $\varphi\left(p_{j}\right)$ respects the parabolic structure, i.e. maps each $V_{k}^{j}$ into itself. Furthermore, let $\mathcal{E} n d_{\text {iso }}(E)$ denote the sheaf of locally isomonodromic endomorphisms, which are by definition those parabolic endomorphisms $\varphi$ whose value $\varphi\left(p_{j}\right)$ at $p_{j}$ lies in the adjoint orbit of the residue of the connection in the Lie algebra $\mathfrak{g l}(m)$; in different terms, $\varphi\left(p_{j}\right)$ maps each $V_{k}^{j}$ into $V_{k+1}^{j}$. By Condition 1.4, the residue at $p_{j}$ is regular diagonal in the trivialisation $v_{1}^{j}, \ldots, v_{m}^{j}$; the parabolic endomorphisms are the ones whose value at $p_{j}$ is a lower-triangular matrix in this basis, and the locally isomonodromic endomorphisms are the ones whose value at $p_{j}$ is a strictly lower-triangular matrix in this basis. Denote by $\mathbf{H}^{i}$ the $i$-th hypercohomology of a sheaf complex. The infinitesimal deformations of the integrable connection $(E, D)$ (without changing the eigenvalues of the residues) are then described by the first hypercohomology $\mathbf{H}^{1}(\mathcal{C})$ of the two-term complex

$$
\mathcal{E} n d_{\text {par }}(E) \stackrel{\operatorname{ad}_{D}}{\longrightarrow} \Omega^{1}(P) \otimes \mathcal{E} n d_{\text {iso }}(E),
$$

where $\operatorname{ad}_{D}$ denotes the action of $D$ on endomorphisms. Write $\nabla=$ $D+\bar{\partial}^{E}$ for the differential geometric flat connection associated to the couple $(E, D)$. We denote by $\mathrm{d}_{\nabla}$ the exterior derivative induced by $\nabla$ on $E$-valued differential forms, and extend it to every bundle functorially associated to $E$. If $(E, D)$ is stable, then there exists a unique 
harmonic metric $h$ on $E$ : this is a Hermitian metric satisfying a secondorder non-linear partial differential equation, that we do not spell out here; for details see e.g. Biq97. Let us denote by ${ }^{*}$ the operation of taking adjoint of an endomorphism of $E$ (or formal adjoint of a differential operator on $E$ ). Set $\Delta=\mathrm{d}_{\nabla} \mathrm{d}_{\nabla}^{*}+\mathrm{d}_{\nabla}^{*} \mathrm{~d}_{\nabla}$ for the associated Laplace operator on differential forms, and call sections annihilated by $\Delta$ harmonic forms. Let us endow the base $\mathbf{P}^{1} \backslash P$ with a metric which looks like the Poincaré metric near the punctures. The elements of $\mathbf{H}^{1}(\mathcal{C})$ are then represented by endomorphism-valued $L^{2}$ harmonic 1-forms. Furthermore, by Proposition 6.1 of [IIS06], cup product on hypercohomology induces a natural complex symplectic structure on $\mathcal{M}$.

The hypercohomology long exact sequence for (C) reads

$$
\begin{aligned}
& 0 \longrightarrow \mathbf{H}^{0}(\mathcal{C}) \longrightarrow H^{0}\left(\mathcal{E} n d_{\mathrm{par}}(E)\right) \stackrel{H^{0}(D)}{\longrightarrow} H^{0}\left(\boldsymbol{\Omega}^{1}(P) \otimes \mathcal{E} n d_{\text {iso }}(E)\right) \longrightarrow \\
& \longrightarrow \mathbf{H}^{1}(\mathcal{C}) \longrightarrow H^{1}\left(\mathcal{E} n d(E)_{\mathrm{par}}\right) \stackrel{H^{1}(D)}{\longrightarrow} H^{1}\left(\boldsymbol{\Omega}^{1}(P) \otimes \mathcal{E} n d_{i s o}(E)\right) \longrightarrow \\
&(10) \quad \longrightarrow \mathbf{H}^{2}(\mathcal{C}) \longrightarrow 0 .
\end{aligned}
$$

The maps $H^{i}(D)$ are induced by $D$ on the corresponding cohomology spaces. Setting

$$
\begin{aligned}
& C=\operatorname{coker}\left(H^{0}(D)\right) \\
& K=\operatorname{ker}\left(H^{1}(D)\right),
\end{aligned}
$$

there follows a short exact sequence for the space of infinitesimal deformations:

$$
0 \longrightarrow C \longrightarrow \mathbf{H}^{1}(\mathcal{C}) \longrightarrow K \longrightarrow 0
$$

Consider $\mathcal{P}_{d}\left(\left\{\beta_{k}^{j}\right\}\right)$, the moduli space of $\beta$-stable parabolic bundles of degree $d=(1-n) m(m-1) / 2$, defined in [MS80]. The moduli space $\mathcal{M}$ admits a Zariski open subset $\mathcal{M}^{0}$ where the underlying parabolic bundle $E$ is stable. Notice that in principle for some choices of parabolic weights $\left\{\beta_{k}^{j}\right\}$ it might happen that there are no stable underlying bundles, i.e. $\mathcal{M}^{0}$ may be empty. In the particular case of rank 2 bundles with 4 parabolic points, this question is analyzed in Section 4 of [LSS10]. Namely, it is proved there that there exists a non-empty set of parabolic weights (given by some explicit inequalities) such that the generic quasi-parabolic structure with these weights is stable. It would be interesting to show a similar statement in the general case too; in the following proposition we tacitly assume this is the case.

Proposition 2.1. The tangent of the forgetful map

$$
\mathcal{M}^{0} \longrightarrow \mathcal{P}_{d}\left(\left\{\beta_{k}^{j}\right\}\right)
$$


mapping $(E, D) \in \mathcal{M}^{0}$ to $E$ is the map

$$
\mathbf{H}^{1}(\mathcal{C}) \longrightarrow K
$$

of (13). Equivalently, the term $C$ in (13) corresponds to infinitesimal modifications of the logarithmic connection on a fixed parabolic bundle E.

Proof. Let $(\alpha, \beta) \in \mathbf{H}^{1}(\mathcal{C})$ be a class in the tangent space at $(E, D)$ of $\mathcal{M}^{0}$ with $\alpha \in H^{0}\left(\Omega^{1}(P) \otimes \mathcal{E} n d_{\text {iso }}(E)\right)$ and $\beta \in H^{1}\left(\mathcal{E} n d_{\text {par }}(E)\right)$ and consider the associated infinitesimal deformation $\left(E_{\varepsilon}, D_{\varepsilon}\right)$. Then $E_{\varepsilon}$ is the holomorphic bundle with $\bar{\partial}$-operator equal to $\bar{\partial}^{E}+\varepsilon \beta$ and $D_{\varepsilon}=$ $D+\varepsilon \alpha$. The cocycle-condition says that $D_{\varepsilon}$ is a logarithmic connection on $E_{\varepsilon}$. Notice that (15) maps $(\alpha, \beta)$ to $\beta$. Therefore, to show that it is the tangent of (14) which forgets the connection $D$ there only remains to prove that for $\beta$-stable $E$ the tangent space $T_{E} \mathcal{P}_{d}\left(\left\{\beta_{k}^{j}\right\}\right)$ is equal to $K$. This is essentially the content of Theorem 5.2 of [MS80].

We now come back to algebraic considerations; in what follows we do not use the stability condition on $E$ any more. Let us next show the duality statement which will be of fundamental importance in the rest of the paper.

Lemma 2.2. For all $(E, D) \in \mathcal{M}$ Serre duality induces a C-vector space isomorphism $K^{\vee} \cong C$.

Here and in all what follows, we use the standard bilinear form

$$
\langle A, B\rangle=\frac{1}{m} \operatorname{tr}\left(A^{t} B\right),
$$

where $A^{t}$ stands for the transpose of $A$, to identify the Lie algebra $\mathfrak{g l}(m)$ with its dual. It also induces orthogonal projection operators to all vector subspaces of $\mathfrak{g l}(m)$.

Proof. Let us compute the dual

$$
C^{-1} \stackrel{\operatorname{ad}_{D^{t}}}{\longrightarrow} C^{0}
$$

of $(\underline{\mathcal{C}})$, where the two non-zero terms are in degree -1 and 0 , and $D^{t}$ stands for the transpose of $D$. Observe that since duality changes the sign of the weights it inverts their order, so $D^{t}$ will be a parabolic map in $(\hat{\mathcal{C}})$. Let us determine the sheaves $C^{i}$. The dual of the sheaf $\mathcal{E} n d(E)$ is clearly $\mathcal{E} n d(E)$ itself, so away from the singular points both $C^{-1}$ and $C^{0}$ clearly coincide with $\mathcal{E} n d(E)$. Consider a singular point $p \in D$ and fix an arbitrary trivialisation of $E$ in a neighborhood of $p$ in which $\operatorname{res}_{p}(D)$ is diagonal. As we have seen, with respect to such a trivialisation $\mathcal{E}_{n d_{\text {par }}}(E)$ is the sheaf of endomorphisms of $E$ vanishing above the 
diagonal. Since the dual of the vanishing condition is having a simple pole, it follows that $C^{0}$ consists of local meromorphic endomorphismvalued 1 -forms near $p$ whose coefficients strictly above the diagonal have a pole of order at most 1 , and all other coefficients are regular. Similarly, since local sections of $\Omega^{1}(P) \otimes \mathcal{E} n d_{\text {iso }}(E)$ near $p$ are local meromorphic 1-forms with strictly lower-triangular residue, it follows that $C^{-1}$ is composed of local holomorphic endomorphisms whose value at $p$ is upper triangular, in other words whose evaluation on the strictly lower-triangular coefficients vanishes. Now, one notices that $C^{-1}$ and $C^{0}$ are the transposes of $\mathcal{E} n d_{\text {par }}(E)$ and $\Omega^{1}(P) \otimes \mathcal{E} n d_{\text {iso }}(E)$. This, together with the fact that the map in $\hat{\mathcal{C}}$ is the transpose of $D$, shows that transposition gives an isomorphism of complexes between $\hat{\mathcal{C}}[-1]$ and $\mathcal{C}$, so Serre duality implies the statement.

Hence, Lemma 2.2 together with (10) exhibits $\mathbf{H}^{1}(\mathcal{C})$ as the extension of two vector spaces of the same dimension:

$$
0 \longrightarrow C \longrightarrow \mathbf{H}^{1}(\mathcal{C}) \longrightarrow K \longrightarrow 0
$$

Let us show that the two factors of this extension are complex conjugate to each other. We first construct an anti-linear map from $C$ to $K$. An element of $C$ is a class of global sections of $\Omega^{1}(P) \otimes \mathcal{E} n d_{i s o}(E)$, modulo the image of the map $H^{0}(D)$ induced by the connection on global sections of $\mathcal{E} n d(E)$. Let $\alpha^{\prime} \mathrm{d} z$ represent such a class. Recall that we denote by $h$ the harmonic metric for the integrable connection and by * the operation of taking adjoint with respect to $h$. Then, one has coker $\left(H^{0}(D)\right) \cong \operatorname{ker}\left(H^{0}(D)^{*}\right)$, therefore there exists a unique global section $\alpha \mathrm{d} z \in \operatorname{ker}\left(H^{0}(D)^{*}\right)$ such that $\alpha \mathrm{d} z=\alpha^{\prime} \mathrm{d} z+D f$ for some $f \in H^{0}(\mathcal{E} n d(E))$. Then, since $\alpha \mathrm{d} z$ is of type $(1,0)$, it follows that

$$
\begin{aligned}
\Delta(\alpha \mathrm{d} z) & =\mathrm{d}_{\nabla}^{*}\left(\bar{\partial}^{E} \alpha \mathrm{d} z\right)+\mathrm{d}_{\nabla}\left(D^{*} \alpha \mathrm{d} z\right) \\
& =\nabla\left(D^{*} \alpha \mathrm{d} z\right) \\
& =D\left(D^{*} \alpha \mathrm{d} z\right) \\
& =0 .
\end{aligned}
$$

The second equality in this sequence holds because $\alpha \mathrm{d} z$ is by assumption a global holomorphic form, the third one follows from the map

$$
H^{0}(D)^{*}: H^{0}\left(\boldsymbol{\Omega}^{1}(P) \otimes \mathcal{E}_{n} d_{i s o}(E)\right) \longrightarrow H^{0}(\mathcal{E} n d(E)),
$$

and the last one is a consequence of the assumption $\alpha \mathrm{d} z \in \operatorname{ker}\left(H^{0}(D)^{*}\right)$. Hence, $\alpha \mathrm{d} z$ is an endomorphism-valued global harmonic (1,0)-form representing the class of $\alpha^{\prime} \mathrm{d} z$. Take the adjoint of the endomorphism with respect to $h$ and the complex conjugate 1 -form: $\alpha^{*} \mathrm{~d} \bar{z}$. According to the $L^{2}$ Dolbeault resolution (Lemma 9.1 and Theorem 5.1, Biq97]) 
it defines an element $\left[\alpha^{*} \mathrm{~d} \bar{z}\right]$ of $H^{1}\left(\mathbf{P}^{1}, \mathcal{E} n d(E)\right)$. Since $\Delta$ is a real operator, we deduce that

$$
\begin{aligned}
0=\Delta\left(\alpha^{*} \mathrm{~d} \bar{z}\right) & =\mathrm{d}_{\nabla}^{*}\left(D \alpha^{*} \mathrm{~d} \bar{z}\right)+\nabla\left(\left(\bar{\partial}^{E}\right)^{*} \alpha^{*} \mathrm{~d} \bar{z}\right) \\
& =\mathrm{d}_{\nabla}^{*}\left(D \alpha^{*} \mathrm{~d} \bar{z}\right),
\end{aligned}
$$

where the second equality follows from $\bar{\partial}^{E} \alpha \mathrm{d} z=0$. By irreducibility of $D$, the 2 -form $D \alpha^{*} \mathrm{~d} \bar{z}$ must be trivial, i.e. the cohomology class $\left[\alpha^{*} \mathrm{~d} \bar{z}\right] \in H^{1}\left(\mathbf{P}^{1}, \mathcal{E} n d(E)\right)$ is in $\operatorname{ker}\left(H^{1}(D)\right)=K$. Call this cohomology class $\varsigma([\alpha \mathrm{d} z])$. Clearly, $\varsigma$ is then an anti-linear map from $C$ to $K$. In the next paragraph we will see that it is bijective.

The map $\varsigma$ can be extended to define a conjugation on the whole tangent space $\mathbf{H}^{1}(\mathcal{C})$. Indeed, by Theorem 12.6 of [Biq97 the hypercohomology $\mathbf{H}^{1}(\mathcal{C})$ is isomorphic to the first $L^{2}$-cohomology of $\nabla$ acting on endomorphisms of $E$ with respect to $h$, or said differently, to the $L^{2}$-kernel of the Laplacian $\Delta$ acting on endomorphisms. Let $\alpha \mathrm{d} z+\beta \mathrm{d} \bar{z}$ be the endomorphism-valued $L^{2}$ harmonic 1-form representing a given class $a \in \mathbf{H}^{1}(\mathcal{C})$. Since the Laplacian is a real operator, the 1 -form $\beta^{*} \mathrm{~d} z+\alpha^{*} \mathrm{~d} \bar{z}$ is also harmonic, hence it represents a class in $\mathbf{H}^{1}(\mathcal{C})$. We declare this class to be $\varsigma(a)$. This map is clearly involutive, in particular bijective.

The conjugation $\varsigma$ coupled with the symplectic structure induces a skew-Hermitian form $g$ on $\mathbf{H}^{1}(\mathcal{C})$ as follows. Let $\alpha_{j} \mathrm{~d} z+\beta_{j} \mathrm{~d} \bar{z}$ be the harmonic representatives of the classes $a_{j} \in \mathbf{H}^{1}(\mathcal{C})$ for $j \in\{1,2\}$. Then $g$ is defined by the formula

$$
\begin{aligned}
g\left(a_{1}, a_{2}\right) & =i a_{1} \cup \varsigma\left(a_{2}\right) \\
& =i \int_{\mathbf{P}^{1}} \operatorname{tr}\left(\alpha_{1} \mathrm{~d} z \wedge \alpha_{2}^{*} \mathrm{~d} \bar{z}+\beta_{1} \mathrm{~d} \bar{z} \wedge \beta_{2}^{*} \mathrm{~d} z\right) .
\end{aligned}
$$

Clearly, $C$ and $K$ are orthogonal complements of each other with respect to this metric. Moreover, the form $i g$ is positive definite on $C$ and negative definite on $K$. In particular, we deduce the orthogonal decomposition of vector spaces

$$
\begin{aligned}
\mathbf{H}^{1}(\mathcal{C}) & =C \oplus K \\
& =H^{1,0} \oplus H^{0,1} .
\end{aligned}
$$

which is just the decomposition of harmonic 1-forms according to type. The second line of this decomposition together with $\varsigma$ define a weight 1 Hodge structure on the tangent of $\mathcal{M}$, and the Hermitian metric (16) induces a polarisation on it. In the next subsection we show that it admits the desired property. 
2.2. Characterisation of deformations of the Fuchsian equation. In this subsection, we show that the Hodge structure defined in the previous subsection satisfies the property claimed in Theorem 1.6. In all this part, we consider a point $\left(E_{\mathcal{L}}, D_{\mathcal{L}}\right) \in \mathcal{E}$, induced by the Fuchsian equation $\mathcal{L}$.

First, notice that it is sufficient to show that for any Fuchsian equation $\mathcal{L}$ with the right exponents, the parabolic bundle $E_{\mathcal{L}}$ is always the same. Indeed, as we have seen in Subsection 2.1, this then implies that an infinitesimal deformation of Fuchsian equations always lies inside the $(1,0)$-part $C$ of the Hodge structure (at least in the case where this parabolic bundle is stable). In different terms, we then have $T_{D_{\mathcal{L}}} \mathcal{E} \subset C$. On the other hand, by Lemma 2.2 and (8) the dimensions of the vector subspaces $T_{D_{\mathcal{L}}} \mathcal{E}$ and $C$ of $T_{D_{\mathcal{L}}} \mathcal{M}$ are both of dimension $e$, so they must agree.

Therefore, suppose we are given any polynomials $G_{1}, \ldots G_{m}$ in the variable $z$ such that $\operatorname{deg}\left(G_{k}\right) \leq k(n-1)$ and that the exponents of the corresponding equation $\mathcal{L}$ at any puncture $p_{j}$ are the numbers $\mu_{1}^{j}, \ldots, \mu_{m}^{j}$ we fixed in advance. It is clear that the lattices (3) and (6) are independent of the choice of such polynomials, just as the gluing matrices between them. On the other hand, the residue at any $p_{j}$ of $D_{\mathcal{L}}$ is equal to a modified companion matrix $A\left(p_{j}\right)$ where $A$ is defined in (5). In particular, for any $1 \leq k \leq m$ its $\mu_{k}^{j}$-eigenspace only depends on the residue, and so is also independent of the polynomials $G_{k}$ themselves. However, the eigenvalues at $p_{j}$ are assumed to be different, and the parabolic structure of $E$ is induced by the eigenvectors of the residue; hence, the parabolic structure is independent of the polynomials $G_{k}$ in the same trivialisation which was already seen to be independent of the choice of $G_{k}$. To sum up, the underlying parabolic bundle $E_{\mathcal{L}}$ is independent of the choice of the polynomials $G_{k}$ satisfying the property that the exponents of $\mathcal{L}$ at all $p^{j}$ are $\mu_{1}^{j}, \ldots, \mu_{m}^{j}$.

2.3. Lagrangian property. Let us denote by $I$ and $J$ the de Rham and Dolbeault complex structures on $\mathcal{M}$ respectively, and set $K=I J$ [Hit87. Further, we denote by $\omega_{I}, \omega_{J}, \omega_{K}$ the associated symplectic structures and set $\Omega_{I}=-\omega_{J}+i \omega_{K}$. We then have:

Corollary 2.3. $\mathcal{E}$ is a complex algebraic Lagrangian submanifold of $\mathcal{M}$ with respect to the holomorphic symplectic form $\Omega_{I}$.

Remark 2.4. The Lagrangian property is proved independently in the recent work [Aid] by Jonathan Aidan. His proof relies on matrix commutator computations, hence is different from ours. 
Proof. Let us consider the canonical family

$$
\left(E_{\mathcal{L}}, D_{\mathcal{L}}\right) \longrightarrow \mathcal{E}
$$

of logarithmic connections on $\mathbf{P}^{1}$ parameterised by $\mathcal{E}$. By construction $(3,4,5,6)$ the bundle $E_{\mathcal{L}}$ is independent of $\mathcal{L}$ and the connection matrix of $D_{\mathcal{L}}$ with respect to the independent trivialisation depends algebraically on the coefficients of the polynomials $G_{1}, \ldots, G_{m}$. It follows that the family (18) is an algebraic family. By the universal property of $\mathcal{M}$, the inclusion map $\mathcal{E} \hookrightarrow \mathcal{M}$ is then algebraic.

Recall that the tangent space of $\mathcal{M}$ is identified with endomorphismvalued $L^{2}$ harmonic 1-forms. For such 1-forms $\phi_{1}, \phi_{2}$ the holomorphic symplectic form of the de Rham moduli space can be written as

$$
\Omega_{I}\left(\phi_{1}, \phi_{2}\right)=\int_{\mathbf{C}} \operatorname{tr}\left(\phi_{1} \wedge \phi_{2}\right)
$$

Clearly this quantity vanishes if both $\phi_{1}$ and $\phi_{2}$ are of type $(1,0)$.

\section{Appendix: The proof of Proposition 1.2}

Proof. Suppose there exists a gauge transformation $g \in \mathcal{H}$ om $\left(E_{\mathcal{L}_{1}}, E_{\mathcal{L}_{2}}\right)$ mapping $D_{\mathcal{L}_{1}}$ into $D_{\mathcal{L}_{2}}$. In the decompositions (7) of $E_{\mathcal{L}_{1}}$ and $E_{\mathcal{L}_{2}}, g$ can be written as a matrix whose entry in the $k$-th row and $l$-th column is a global holomorphic section of the sheaf $\mathcal{O}((k-l)(n-1))$. It follows that the matrix of $g$ is lower triangular, and that the entries on the diagonal are global sections of the trivial holomorphic line bundle over $\mathbf{P}^{1}$, hence constants. For $j=1,2$ let us write on the affine part $\mathbf{C}$ of $\mathbf{P}^{1}$ away from infinity the expressions

$$
D_{\mathcal{L}_{j}}=\mathrm{d}^{1,0}-\frac{A_{j}(z)}{\psi(z)} \mathrm{d} z .
$$

It is then a well-known fact that the action of $g$ on $D_{\mathcal{L}_{1}}$ is

$$
g \cdot\left(D_{\mathcal{L}_{1}}\right)=\mathrm{d}^{1,0}-\frac{g^{-1} A_{1}(z) g}{\psi(z)} \mathrm{d} z-g^{-1} d^{1,0} g
$$

It follows from the above observations that $g^{-1} d^{1,0} g$ is strictly lower triagular. In particular, the entries on and above the diagonal in the matrices $A_{1}$ and $A_{2}$ must agree.

Let us first consider the case $m=2$. Here, one has

$$
g=\left(\begin{array}{cc}
g_{11} & 0 \\
g_{21} & g_{22}
\end{array}\right),
$$


where $g_{21}$ is a global section of $\mathcal{O}(n-1)$ and $g_{11}, g_{22}$ are constants with $g_{11} g_{22} \neq 0$, and the inverse of this matrix is

$$
g^{-1}=\frac{1}{g_{11} g_{22}}\left(\begin{array}{cc}
g_{22} & 0 \\
-g_{21} & g_{11}
\end{array}\right) \text {. }
$$

Furthermore, the matrices of the equations are

$$
A_{j}=\left(\begin{array}{cc}
0 & 1 \\
G_{2}^{j} & G_{1}^{j}+\psi^{\prime}
\end{array}\right)
$$

where $G_{2}^{j}, G_{1}^{j}$ are the coefficients of $\mathcal{L}_{j}$. Straightforward matrix multiplication yields

$$
g^{-1} A_{1}(z) g=\frac{1}{g_{11} g_{22}}\left(\begin{array}{cc}
g_{21} g_{22} & g_{22}^{2} \\
* & *
\end{array}\right)
$$

By the above, the terms in the first row must be equal to 0 and 1 respectively. We infer that $g_{21}=0$ and $g_{22}=g_{11}$, hence $g$ is a multiple of the identity.

We now come to the general case. As the computations are more involved but of the same spirit, we only sketch the proof. The matrix $g$ is equal to

$$
g=\left(\begin{array}{cccc}
g_{11} & 0 & \ldots & 0 \\
g_{21} & g_{22} & \ldots & 0 \\
\vdots & & \ddots & \vdots \\
g_{m 1} & g_{m 2} & \ldots & g_{m m}
\end{array}\right)
$$

and its inverse is of the form

$$
g^{-1}=\frac{1}{g_{11} g_{22} \cdots g_{m m}}\left(\begin{array}{cccc}
g_{22} \cdots g_{m m} & 0 & \cdots & 0 \\
* & g_{11} g_{33} \cdots g_{m m} & \cdots & 0 \\
\vdots & & \ddots & \vdots \\
* & * & \cdots & g_{11} \cdots g_{m-1, m-1}
\end{array}\right)
$$


One has

$$
\begin{aligned}
& g^{-1} A_{1}(z) g=\frac{1}{g_{11} g_{22} \cdots g_{m m}} . \\
& \begin{array}{c}
\left(\begin{array}{ccccc}
g_{22} \cdots g_{m m} & 0 & \cdots & 0 \\
* & g_{11} g_{33} \cdots g_{m m} & \cdots & \vdots \\
\vdots & & \ddots & 0 \\
* & * & \cdots & g_{11} \cdots g_{m-1, m-1}
\end{array}\right) \\
\left(\begin{array}{ccccc}
g_{21} & g_{22} & 0 & \cdots & 0 \\
g_{21} \psi^{\prime}+g_{31} & g_{22} \psi^{\prime}+g_{32} & g_{33} & \cdots & \vdots \\
\vdots & & \ddots & \ddots & 0 \\
* & & & g_{m-1, m-1} \psi^{\prime}+g_{m, m-1} & g_{m m} \\
* & \ldots & & * & *
\end{array}\right) .
\end{array}
\end{aligned}
$$

As the entries on and above the diagonal in this product have to be equal to those of the modified companion matrix $A_{2}(z)$, we deduce as before that $g_{11}=g_{22}=\cdots=g_{m m}$ and $g_{21}=g_{32}=\cdots=g_{m, m-1}=0$. It follows that right below the diagonal all the entries of the matrix $g^{-1} d^{1,0} g$ vanish. Considering now the first sub-diagonal in the product above, we deduce that $g_{31}=g_{42}=\cdots=g_{m, m-2}=0$. It follows that on the second sub-diagonal of the matrix $g^{-1} d^{1,0} g$ all the entries vanish. Continuing this argument, we eventually obtain that all the $g_{k l}$ for $k>l$ must vanish. This concludes the proof.

\section{REFERENCES}

[Aid] Jonathan Aidan. Propriétés symplectiques de l'espace des équations differentielles dans l'espace des systèmes logarithmiques. $\mathrm{PhD}$ thesis, Université Pierre et Marie Curie, Paris. In preparation.

[BB04] Olivier Biquard and Philip Boalch. Wild non-abelian Hodge theory on curves. Compositio Mathematica, 140(1):179-204, 2004.

[BD05] Alexander Beilinson and Vladimir Drinfeld. Opers. arXiv:math/0501398, 2005.

[Biq97] Olivier Biquard. Fibrés de Higgs et connexions intégrables: le cas logarithmique (diviseur lisse). Annales scientifiques de l'École Normale Supérieure, 30(4):41-96, 1997.

[Del70] Pierre Deligne. Équations différentielles à points singuliers réguliers, volume 163 of Lecture Notes in Mathematics. Springer-Verlag, 1970.

[Hit87] Nigel J. Hitchin. The self-duality equations on a Riemann surface. Proceedings of the London Mathematical Society, 55(3):59-126, 1987.

[Hit92] Nigel J. Hitchin. Lie groups and Teichmüller space. Topology, 31(3):449473, 1992.

[IIS06] Michi-Aki Inaba, Katsunori Iwasaki, and Masa-Hiko Saito. Moduli of stable parabolic connections, Riemann-Hilbert correspondence and geometry 
of Painlevé equation of type VI, part I. Publications of the Research Institute for Mathematical Sciences, 42(4), 2006.

[Ina06] Michi-Aki Inaba. Moduli of parabolic connections on a curve and RiemannHilbert correspondence. arXiv:math/0602004, 2006.

[Inc26] E. L. Ince. Ordinary Differential Equations. Dover Publications, New York, 1926.

[Kat96] Nicholas M. Katz. Rigid Local Systems. Number 139 in Annals of Mathematics Studies. Princeton University Press, 1996.

[LSS10] Frank Loray, Masa-Hiko Saito, and Carlos Simpson. Foliations on the moduli space of rank two connections on the projective line minus four points. arXiv:1012.3612, 2010.

[MS80] V. B. Mehta and C. S. Seshadri. Moduli of vector bundles on curves with parabolic structures. Mathematische Annalen, 248:205-239, 1980.

[Nit93] Nitin Nitsure. Moduli of semistable logarithmic connections. Journal of the American Mathematical Society, 6:597-609, 1993.

[SS] M-H. Saito and Sz. Szabó. Apparent singularities and canonical coordinates for moduli of connections. In preparation.

[Sza08] Szilárd Szabó. The extension of a Fuchsian equation onto the projective line. Acta Scientiarum Mathematicarum (Szeged), 74:557-564, 2008. 